\title{
Beauty Production with ALICE at the LHC
}

\author{
Erin F. Gauger for the ALICE Collaboration \\ The University of Texas at Austin, Austin TX 78712, USA, \\ erin.gauger@cern.ch
}

\begin{abstract}
In this manuscript, various beauty production measurements using the ALICE detector will be presented. We will show new measurements of non-prompt $\mathrm{D}^{0}$ mesons in pp collisions at $\sqrt{s}=5.02 \mathrm{TeV}$ and beauty-tagged jet production in $\mathrm{p}-\mathrm{Pb}$ collisions at $\sqrt{s_{\mathrm{NN}}}=5.02 \mathrm{TeV}$. The $R_{\mathrm{AA}}$ of beauty-hadron decay electrons in central $\mathrm{Pb}-\mathrm{Pb}$ collisions and the $v_{2}$ of beauty-hadron decay electrons in semi-central $\mathrm{Pb}-\mathrm{Pb}$ collisions at $\sqrt{s_{\mathrm{NN}}}=5.02 \mathrm{TeV}$ will also be discussed.
\end{abstract}

Keywords: heavy quarks, beauty quarks, nuclear modification factor

\section{Introduction}

In hadronic collisions, beauty quarks are produced early via hard-scattering processes with large momentum transfer. Because of this early production, the beauty quark is an excellent probe of the Quark-Gluon Plasma (QGP) [1|2 formed in heavy-ion collisions. Traveling through the QGP, beauty quarks interact with other partons via collisional and radiative processes and lose energy. The beauty quark $\left(m_{\mathrm{b}} \simeq 4.18 \mathrm{GeV} / c^{2}\right.$ [4] $)$ is expected to lose less energy than lighter quarks, since collisional processes depend on the mass of the particle and the dead cone effect [3] would hamper radiative energy loss. Since charm $\left(m_{\mathrm{c}} \simeq 1.27 \mathrm{GeV} / c^{2}\right.$ [4] $)$ and beauty quarks are both produced early in the collision but have different masses, it is useful to compare beauty and charm measurements to test our understanding of mass-dependent energy loss in heavy-ion collisions.

Measurements of beauty production in pp collisions are important to test perturbative QCD (pQCD) calculations, as well as to provide the baseline for $\mathrm{Pb}-\mathrm{Pb}$ measurements. In $\mathrm{p}-\mathrm{Pb}$ collisions, beauty-production measurements are crucial to isolate initial-state and cold nuclear matter effects, both of which would be present in $\mathrm{Pb}-\mathrm{Pb}$ measurements.

\section{Beauty measurements}

In ALICE, beauty production is measured via beauty-hadron decay electrons, non-prompt $\mathrm{D}^{0}$ mesons, and beauty-tagged jets. All three measurements rely on the excellent vertex reconstruction and impact parameter resolution of the ALICE detector to isolate the decay particles and jets from beauty decay. 


\subsection{Beauty-hadron decay electrons}

Roughly $10 \%$ of beauty hadrons decay directly into electronic final states (e.g. $\mathrm{B}^{-} \rightarrow \mathrm{e}+\mathrm{X}$ ), and another $10 \%$ decay to charm hadrons which further decay to electrons (e.g. $\left.\mathrm{B}^{-} \rightarrow \mathrm{D}^{0} \rightarrow \mathrm{e}+\mathrm{X}\right)$ [4]. This high branching ratio coupled with the excellent electron identification of the ALICE detector makes it convenient to study beauty quarks by measuring the production of beauty-hadron decay electrons. Beauty-hadron decay electrons have been measured in $\mathrm{pp}, \mathrm{p}-\mathrm{Pb}$, and $\mathrm{Pb}-\mathrm{Pb}$ collisions [5677.

Electrons from beauty-hadron decays must be separated from other sources of electrons, such as photon conversion, Dalitz decays, and charm-hadron decays. This separation is achieved by exploiting the relatively long decay length of beauty hadrons $\left(\tau_{B} \approx 500 \mu \mathrm{m} / c\right)$ versus non-beauty hadrons (e.g. $\left.\tau_{D}=60-300 \mu \mathrm{m} / c\right)$ [4. The long decay length gives beauty-hadron decay electrons a longer distance of closest approach to the primary vertex $\left(d_{0}\right)$, thus making the beauty-electron $d_{0}$ distribution much wider than that from other sources (Fig. 1. bottom left). The difference in $d_{0}$ shape allows us to fit the electron $d_{0}$ distribution with templates from Monte Carlo simulations in order to extract the beauty-hadron decay electron yield. Once the yields are obtained in both pp and $\mathrm{Pb}-\mathrm{Pb}$ collisions, the nuclear modification factor $R_{\mathrm{AA}}=\frac{\mathrm{d} N_{\mathrm{AA}} / \mathrm{d} p_{\mathrm{T}}}{T_{\mathrm{AA}} * \mathrm{~d} \sigma_{\mathrm{pp}} / \mathrm{d} p_{\mathrm{T}}}$ can be calculated. The pp reference spectrum for the beauty-hadron decay electron $R_{\mathrm{AA}}$ measurement in $\mathrm{Pb}-\mathrm{Pb}$ at $\sqrt{s_{\mathrm{NN}}}=5.02 \mathrm{TeV}$ was obtained by a pQCD-driven scaling of the cross section measured at $\sqrt{s}=7 \mathrm{TeV}\left[5\right.$. In order to obtain a $v_{2}$ measurement, the yield is measured for electrons that lie both in and out of the event plane, and the $v_{2}$ is calculated according to $v_{2}=\frac{1}{R^{2}} \frac{\pi}{4} \frac{N_{\text {in-plane }}-N_{\text {out }- \text { of }- \text { plane }}}{N_{\text {in- } \text { lane }}+N_{\text {out }- \text { of }- \text { plane }}}$, where $N$ refers to the number of electrons measured, and $R$ is the resolution correction for the event plane.

Two beauty-electron measurements in ALICE are $R_{\mathrm{AA}}$ in $0-10 \%$ and $v_{2}$ in $20-40 \%$ central $\mathrm{Pb}-\mathrm{Pb}$ collisions at $\sqrt{s_{\mathrm{NN}}}=5.02 \mathrm{TeV}$. In Fig. 1 top left, the beauty-electron $R_{\mathrm{AA}}$ is shown along with the $R_{\mathrm{AA}}$ of heavy-flavor decay electrons (from both charm and beauty decays). Though the systematic error bars are large, a hint of an increased $R_{\mathrm{AA}}$ of beauty-hadron decay electrons when compared with heavy-flavor electrons at low- $p_{\mathrm{T}}$ is observed. This is consistent with our expectations of the mass dependence of energy loss in the QGP medium. At high $p_{\mathrm{T}}$, the two distributions overlap, in part because at higher momentum, the heavy-flavor electron sample becomes dominated by beauty-hadron decay electrons. Fig. 1 (top right) shows a comparison of the $R_{\mathrm{AA}}$ of beauty-hadron decay electrons with models that include both collisional and radiative energy loss. We see that the theoretical models are in good agreement with data.

Finally, the $v_{2}$ of beauty-hadron decay electrons is shown in Fig. 1 (bottom right). The $v_{2}$ is non-zero; in fact, between $1.3<p_{\mathrm{T}}<4 \mathrm{GeV} / c$, the significance of the measurement for a positive $v_{2}$ is $3.49 \sigma$. This hints that the beauty quark may participate in the collective behavior of the medium. The $v_{2}$ measurement of heavy-flavor decay electrons [8, also shown in Fig. 1] is similar to that of electrons from beauty decays. 

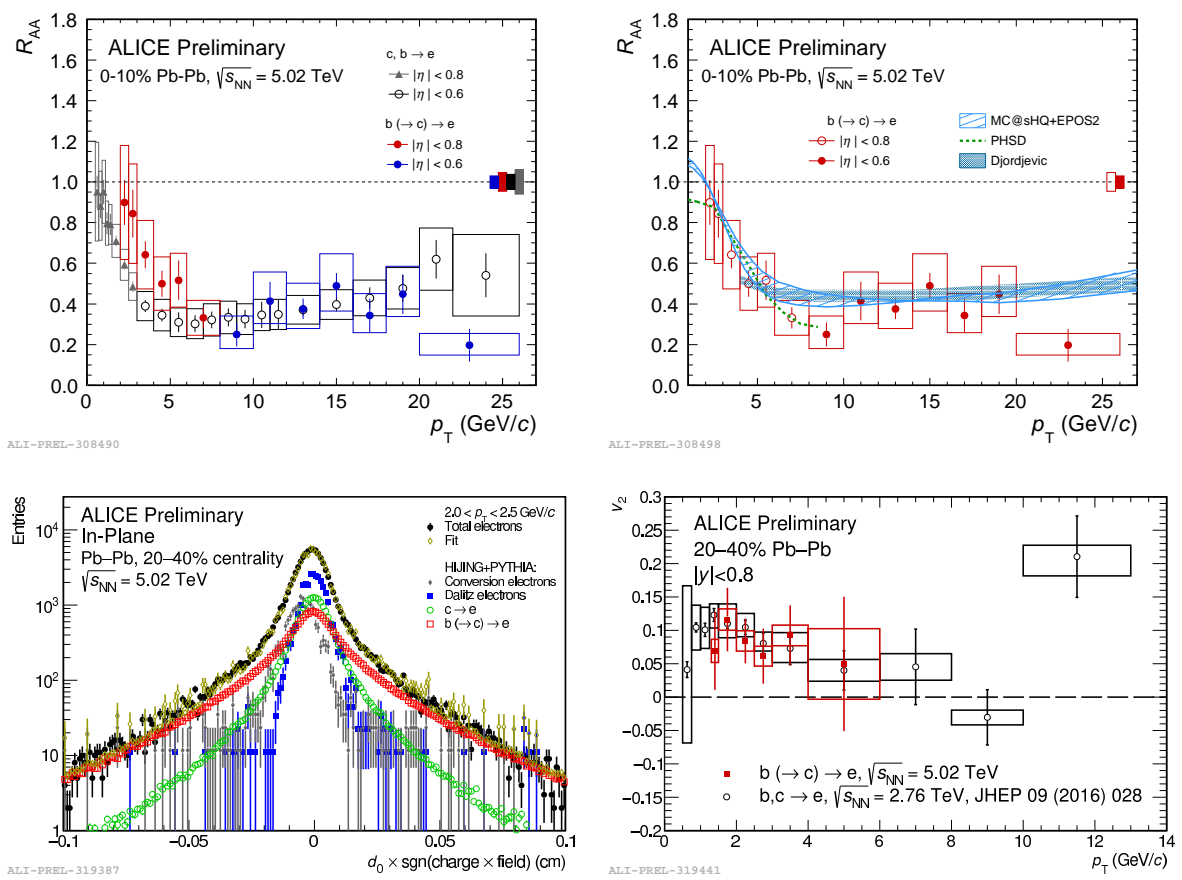

Fig. 1. Various results of beauty-hadron decay electrons in ALICE. The top two panels show the $R_{\mathrm{AA}}$ in $0-10 \% \mathrm{~Pb}-\mathrm{Pb}$ collisions. The bottom-left panel shows an example of a MC template fit of the $d_{0}$ of electrons to extract those from beauty-decay. The bottom-right panel shows the $v_{2}$ of beauty-hadron decay electrons in $20-40 \% \mathrm{~Pb}-\mathrm{Pb}$ collsions.

\section{$2.2 \quad$ Non-prompt $\mathrm{D}^{0}$ mesons}

With the ALICE detector, beauty production is also studied by measuring nonprompt $\mathrm{D}^{0}$ mesons from beauty-hadron decays. The measurement is performed in pp collisions at $\sqrt{s}=5.02 \mathrm{TeV}$. The non-prompt $\mathrm{D}^{0}$ mesons (along with their charge conjugates) are reconstructed via the decay channel to $\mathrm{K}^{-} \pi^{+}$(branching ratio $\sim 3.9 \%)$ and selected by applying various topological requirements, including a cut on the distance between the primary and secondary vertices. The topological selection criteria are optimized using boosted decision trees, allowing us to achieve a high fraction of non-prompt $\mathrm{D}^{0}$ mesons $\left(f_{\text {non-prompt }}\right)$ in our sample. At low- $p_{\mathrm{T}}, f_{\text {non-prompt }}$ almost reaches $95 \%$, an unprecedented purity for this measurement (see Fig. 2). The cross-section of non-prompt $\mathrm{D}^{0}$ mesons is compared with FONLL [10] predictions (pQCD) in Fig. 22 As in previous measurements 9., the two are in agreement, though the measurement lies on the upper edge of the FONLL uncertainty band. 

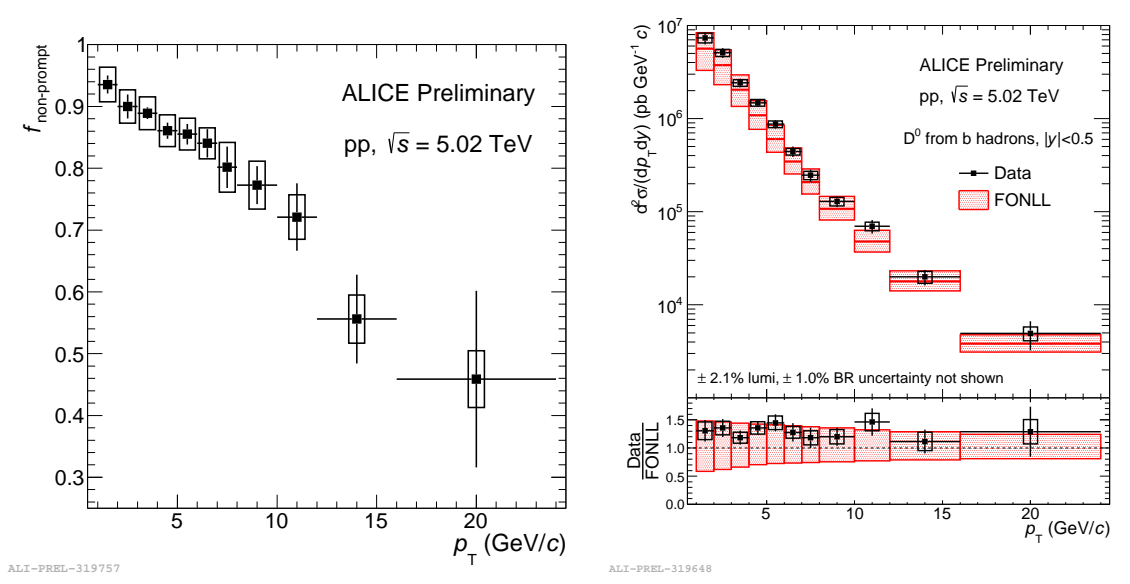

Fig. 2. Left: the fraction of non-prompt to inclusive $D^{0}$ mesons in the sample. Right: The cross section of non-prompt $\mathrm{D}^{0}$ mesons in pp collisions at $\sqrt{s}=5.02 \mathrm{TeV}$ compared to FONLL 10.

\subsection{Beauty-tagged Jets}

A more direct access to the initial parton kinematics is obtained by measuring beauty-tagged jets. This has been done for the first time in ALICE in $\mathrm{p}-\mathrm{Pb}$ collisions at $\sqrt{s_{\mathrm{NN}}}=5.02 \mathrm{TeV}$. Jets are selected using the anti- $k_{\mathrm{T}}$ algorithm 11, and a resolution parameter of $R=0.4$. To achieve a high purity of b-jets in the sample, the long lifetime of beauty hadrons is exploited once more. Jets that contain a three-pronged secondary vertex are selected, and a number of topological requirements are applied to increase the b-jet purity. In particular, a cut is applied on the displacement significance $\left(S L_{x y}>7\right)$ of the secondary vertex. The $S L_{x y}$ is defined as the distance between primary and secondary vertex in the $x y$-plane divided by the resolution of that distance. The results of this measurement are shown in Fig. 3 , where the estimated purity $(\sim 40 \%)$ of beauty-tagged jets in the sample is shown along with the jet cross-section compared POWHEG HVQ and POWHEG dijet EPPS16. We see that both models agree with the measured cross-section.

\section{Conclusions}

In this manuscript the ALICE results on beauty production were discussed with particular focus on beauty-hadron decay electrons in $\mathrm{Pb}-\mathrm{Pb}$ collisions, nonprompt $\mathrm{D}^{0}$ mesons in pp collisions, and beauty-tagged jets in $\mathrm{p}-\mathrm{Pb}$ collisions. All three analyses took advantage of the long lifetime of beauty hadrons to separate the beauty signal from background sources. The non-prompt $\mathrm{D}^{0}$ meson measurement agrees with FONLL predictions, and the beauty-tagged jet measurements 

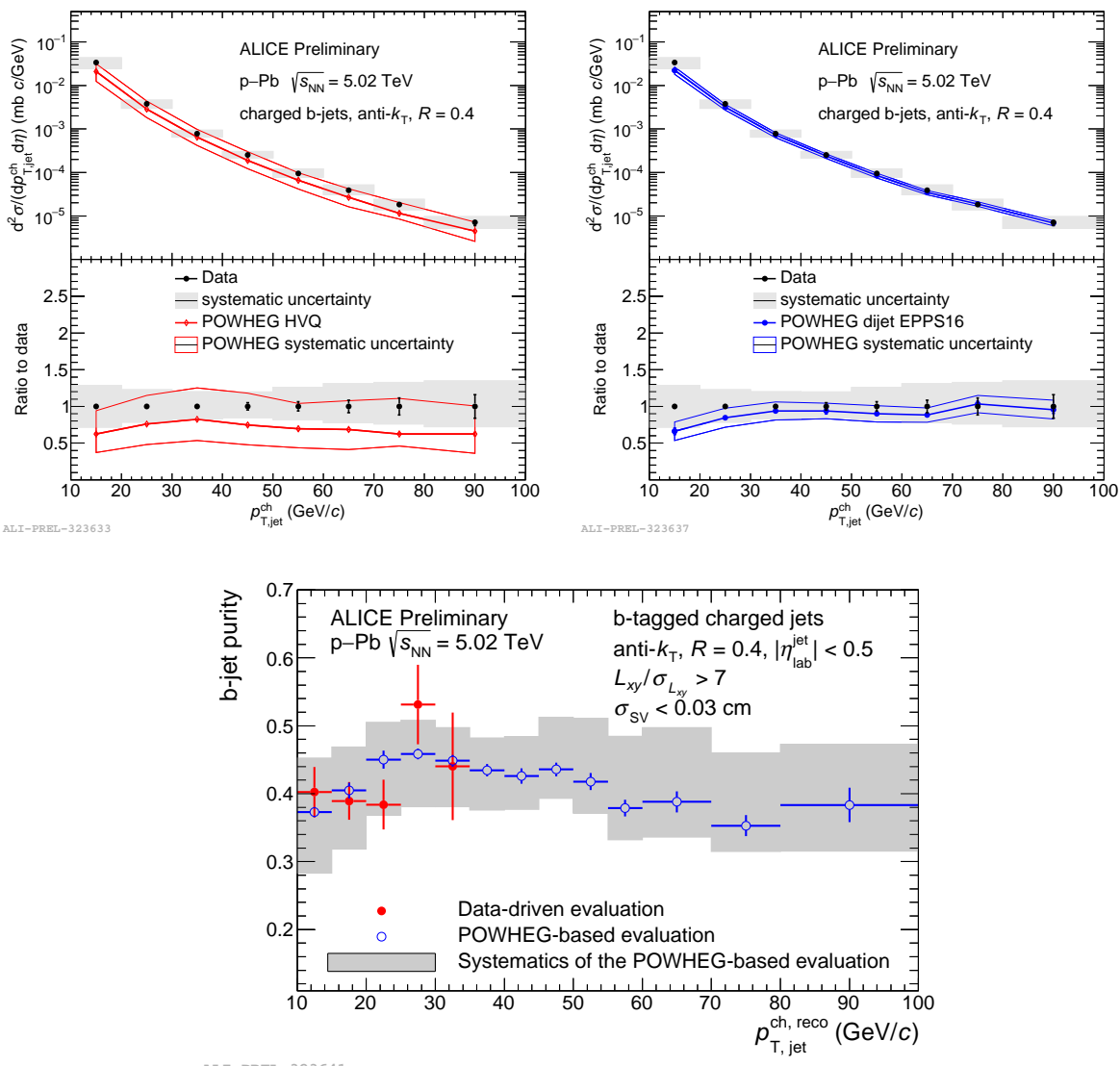

ALI-PREL-323641

Fig. 3. Top left and right: the cross-section of beauty-tagged jets in $\mathrm{p}-\mathrm{Pb}$ collisions compared with POWHEG. Bottom: the purity of beauty jets in the sample.

agree with POWHEG models. In $\mathrm{Pb}-\mathrm{Pb}$ collisions, we see a hint of the massdependent energy loss in the QGP, as well as a non-zero $v_{2}$ for beauty-hadron decay electrons.

Acknowledgements: This work was supported by U.S. Department of Energy Office of Science under contract number de-sc0013391.

\section{References}

1. S. Borsanyi et al., JHEP 09 (2010) 073.

2. A. Bazavov et al., Phys. Rev. D 90094503 (2014).

3. N. Armesto, et al., Phys. Rev. D 69114003 (2004). 
4. M. Tanabashi et al. (Particle Data Group), Phys. Rev. D 98, 030001 (2018) and 2019 update.

5. B. Abelev et al. (ALICE Collaboration), Phys. Lett. B 721 (2013) 13-23.

6. B. Abelev et al. (ALICE Collaboration), Phys. Lett. B 738 (2014) 97.

7. J. Adam et al. (ALICE Collaboration), JHEP 07 (2017) 052.

8. J. Adam et al. (ALICE Collaboration), JHEP 09 (2016) 028.

9. A.M. Sirunyan et al. (CMS Collaboration), Phys. Rev. Lett. 123, 022001 (2019).

10. M. Cacciari, M. Greco and P. Nason, JHEP 9805 (1998) 007 arXiv:hepph/9803400; M. Cacciari, S. Frixione and P. Nason, JHEP 0103 (2001) 006 arXiv:hep-ph/0102134.

11. M. Cacciari, G.P. Salam, and G. Soyez, JHEP 0804 (2008) 063 arXiv:0802.1189 [hep-ph]]. 